\title{
Photomodification of carrier lifetime and diffusivity in AIGaN epitaxial layers
}

\author{
Žydrūnas Podlipskas ${ }^{1, a)}$, Ramūnas Aleksiejūnas ${ }^{1}$, Saulius Nargelas $^{1}$, Jonas Jurkevičius ${ }^{1}$, Jūras \\ Mickevičius ${ }^{1}$, Arūnas Kadyss ${ }^{1}$, Gintautas Tamulaitis ${ }^{1}$, Michael S. Shur ${ }^{2}$, Max Shatalov ${ }^{3}$, Jinwei \\ Yang $^{3}$ and Remis Gaska ${ }^{3}$
}

${ }^{1}$ Institute of Applied Research and Semiconductor Physics Department, Vilnius University, Sauletekis Ave. 9, bld. 3, LT-10222, Vilnius, Lithuania

${ }^{2}$ Department of ECE and CIE, Rensselaer Polytechnic Institute, Troy, New York 12180, USA

${ }^{3}$ Sensor Electronic Technology, Inc., 1195 Atlas Road, Columbia, South Carolina 29209, USA

a) Author to whom correspondence should be addressed. Electronic mail: zydrunas.podlipskas@gmail.com

\begin{abstract}
Nonradiative recombination rate and diffusivity of nonequilibrium carriers were modified by intense laser pulses in AlGaN epilayers with $\mathrm{Al}$ content ranging from 16 to $71 \%$. The epilayers were examined before and after the photomodification using light-induced transient grating and photoluminescence spectroscopy techniques. The photomodification resulted in (i) enhancement of the nonradiative recombination rate and (ii) large changes of the diffusion coefficient of the nonequilibrium carriers, without imposing any macroscopic structural damage to the epilayers. The photomodification effect on the recombination rate was stronger in the layers with higher $\mathrm{Al}$ content indicating the involvement of the $\mathrm{Al}$ atoms in this process. The carrier diffusivity exhibited a rapid initial increase as a consequence of the photomodification followed by a slow decline, as the photomodification duration was increased. The enhancement of the diffusion coefficient of up to 2.4 times was accompanied by $13 \%$ decrease in the carrier lifetime.
\end{abstract}

Keywords: $\mathrm{AlGaN}$ epilayers; nonradiative recombination; diffusion coefficient; recombination enhanced defect reactions; internal quantum efficiency 


\section{Introduction}

AlGaN alloys enable to develop a number of devices, such as deep UV light emitting diodes (LEDs), laser diodes (LDs), solar-blind photodetectors (PDs), and high-power field effect transistors (FET). The efficiency of LEDs and LDs, responsivity of PDs, and cutoff frequency of FETs depend on the nonradiative carrier lifetime and mobility. The values of these parameters in AlGaN are still considerably smaller than those in InGaN, and all recent optimizations of AlGaN growth have not radically narrowed this gap.

An alternative strategy for improving these characteristics could be based on the post-growth treatment of the AlGaN alloys. A particularly interesting approach is to treat the material by creating a high density of nonequilibrium carriers. For example, the injection of electrons into p-AlGaN results in their trapping by neutral $\mathrm{Mg}$ dopants. As a result, the conduction band electrons are not able to recombine via these $\mathrm{Mg}$ levels, and their lifetime and, consequently, diffusion length increase.[1-3] However, this effect is temporary and vanishes at $\sim 300 \mathrm{~K}$ within several days.[1]

Another mechanism of modifications induced by nonequilibrium carriers is the recombination enhanced defect reactions (REDRs),[4,5] which are activated at a rather high carrier density and/or temperature. During the REDR process, the vibrational energy released during the nonradiative recombination can be channeled to a defect center and trigger its transformation, such as diffusion, dissociation, or annihilation. Additional defects can also be formed in this way. Some of them might act as the nonradiative recombination centers decreasing the carrier lifetime and the photoluminescence intensity in the AlGaN alloys.[6]

In this work, we study the photomodification of the carrier lifetime and diffusion coefficient in AlGaN epilayers under intense laser illumination. We use the photoluminescence spectroscopy (PL) and light induced transient grating (LITG) techniques to monitor the effect of the photomodification on the carrier dynamics. We examined the AlGaN layers with $\mathrm{Al}$ content in a wide range from 16 to $71 \%$ and found it being the main factor determining the susceptibility of the epilayer to the photomodification. We show that the experimentally observed PL quenching is caused by an increase in the nonradiative recombination rate, which we attribute to the defect activation/generation via a REDR process.

\section{Material and methods}

Six $0.3-1 \mu \mathrm{m}$ thick uncapped AlGaN epilayers were investigated. The epilayers containing $16 \%$ and $46 \%$ of aluminum were grown using metalorganic chemical vapor deposition (MOCVD) on c-plane $\mathrm{GaN} /$ sapphire and AlN/sapphire templates, respectively. The samples containing 48, 65, 68, and 71\% of the Al molar fraction were grown using the migration-enhanced MOCVD (MEMOCVD ${ }^{\circledR}$ ) on the cplane AlN/sapphire substrates.

The LITG experiments were carried out using a YAG: $\mathrm{Nd}^{+3}$ laser delivering $25 \mathrm{ps}$ pulses at the repetition rate of $10 \mathrm{~Hz}$. Two coherent pulses at the wavelength $\lambda=213 \mathrm{~nm}$ intersecting at the angle $\Theta$ 
on the sample surface created a transient spatially periodic pattern of photoexcited carriers with spacing $\Lambda=\lambda /[2 \sin (\theta / 2)] .[7,8]$ The resulting modulation of the refractive index was proportional to the photoexcited carrier density $N,[7,8]$ this modulation created a transient diffraction grating. The grating decay was monitored by measuring the diffraction efficiency $\eta$ (the ratio between intensities of the diffracted and transmitted beams) of a delayed probe pulse in the transparency region of the sample. The first harmonic $(\lambda=1064 \mathrm{~nm})$ was exploited for probing. Figure 1 depicts typical LITG kinetics in the samples under study. They are non-exponential. We fitted the LITG decay with a biexponential function $\eta(t) \propto N_{1 \mid t=0}^{2} \times \exp \left(-2 t / \tau_{G 1}\right)+N_{2 \mid t=0}^{2} \times \exp \left(-2 t / \tau_{G 2}\right)$, where $\tau_{\mathrm{G} 1}$ and $\tau_{\mathrm{G} 2}$ are the grating decay times of the fast and slow components. The LITG decays due to carrier recombination and diffusion.[7,8] Since localization is important in $\mathrm{AlGaN}$, we interpret the fast and slow decay components as caused by recombination and diffusion of free carriers and recombination of localized carriers, respectively. The short grating decay time $\tau_{\mathrm{G} 1}$ depends on the effective recombination time $\tau_{\text {effl }}$ reflecting both radiative and nonradiative recombination and the ambipolar diffusion coefficient $D:[7,8]$

$$
1 / \tau_{G 1}=1 / \tau_{e f f 1}+4 \pi^{2} D / \Lambda^{2}
$$

The grating period $\Lambda$ was varied from 1.96 to $7.72 \mu \mathrm{m}$ by changing the angle $\Theta$ between the beams recording the grating. Equation (1) was used to simultaneously extract the values of $D$ and $\tau_{\text {effl }}$ from the plot linking the grating decay time and the grating period (see figure 1 and inset therein). The LITG measurements were performed at room temperature.

The photoluminescence of the AlGaN epilayers was studied under quasi-steady-state conditions, and excited by the $5^{\text {th }}$ harmonic $(213 \mathrm{~nm})$ of the Q-switched YAG:Nd laser radiation (pulse duration $4 \mathrm{~ns}$, repetition rate $10 \mathrm{~Hz}$ ). The PL signal was analyzed by a double monochromator (Jobin Yvon HRD-1), detected by an UV-enhanced photomultiplier and processed using a box-car integrator. A closed-cycle helium cryostat ensured the variation and stabilization of temperature in the range from 8 to $300 \mathrm{~K}$. The temperature dependences of the PL band peak shift were exploited to estimate the carrier localization conditions described by the potential fluctuation dispersion parameter $\sigma$, which was extracted, as usual, using a simple model of the carrier (exciton) hopping through the localized states.[9]

Photomodification was performed at room temperature with the number of the laser pulses controlling its duration. The photomodification threshold was determined for every epilayer by taking into account the different light absorption coefficients at $213 \mathrm{~nm}$ (from $3.1 \times 10^{5} \mathrm{~cm}^{-1}$ for $\mathrm{Al}_{0.16} \mathrm{Ga}_{0.84} \mathrm{~N}$ to $1.6 \times 10^{5} \mathrm{~cm}^{-1}$ for $\mathrm{Al}_{0.71} \mathrm{Ga}_{0.52} \mathrm{~N}$ ).[10] All the reported LITG and PL measurements were carried out at the "safe" excitation intensities with carrier densities $N_{\text {ref }}$ and $N_{\mathrm{PL}}$ (estimated using the laser pulse parameters), which were well below the modification threshold $\left(N_{\text {ref }}=3 \times 10^{19} \mathrm{~cm}^{-3}\right.$ for LITG and $N_{\mathrm{PL}}=4 \times 10^{18} \mathrm{~cm}^{-3}$ for PL). Optical micrographs taken before and after the photomodification verified that the photomodification was not related to any optical damage to the sample surface. 


\section{Results}

Figure 2 shows the decrease of the diffraction efficiency $\eta$ in $\mathrm{Al}_{0.71} \mathrm{Ga}_{0.29} \mathrm{~N}$ after 1600 laser pulses (black circles) as a function of the carrier density $N$, which was generated by the photomodifying laser pulse and was estimated from the pulse energy density. Each point of this curve was measured on a separate, previously unmodified sample spot. The efficiency $\eta$ is the ratio of the diffraction efficiencies after and before the photomodification of a particular spot at zero delay time between the grating recording and probe pulses. The carrier density of $N \approx 6 \times 10^{19} \mathrm{~cm}^{-3}$ resulting in a $5 \%$ decrease of $\eta$ was taken as the photomodification threshold. At $N \approx 3 \times 10^{20} \mathrm{~cm}^{-3}, \eta$ decreased by nearly $50 \%$. The exposition of $\mathrm{Al}_{0.71} \mathrm{Ga}_{0.29} \mathrm{~N}$ sample at $N \approx 4 \times 10^{19} \mathrm{~cm}^{-3}$ (just below the threshold) did not impose any significant changes to the sample even after tens of thousands of the laser pulses.

The photomodification-induced changes of the carrier lifetime were assessed by analyzing the decay time $\tau_{\text {effl }}$ of the initial fast component in LITG transient. The ABC model based expression $1 / \tau_{\text {eff } 1}=$ $1 / \tau_{n r 1}+1 / \tau_{\text {rad }}=1 / \tau_{n r 1}+B N$ (neglecting the Auger recombination) was used to separate the radiative and nonradiative components of $\tau_{\text {effl }}$. Here, $\tau_{\mathrm{nr} 1}$ is the nonradiative recombination time, $B$ is the coefficient of radiative bimolecular recombination, and $N$ is the nonequilibrium carrier density.

Two tendencies were revealed: i) the radiative recombination rate was not affected by the photomodification (e.g., $B=9 \times 10^{-11} \mathrm{~cm}^{3} / \mathrm{s}$ remained unchanged after modification of the $\mathrm{Al}_{0.71} \mathrm{Ga}_{0.29} \mathrm{~N}$ layer at $N \approx 3 \times 10^{20} \mathrm{~cm}^{-3}$ with 1600 pulses); ii) $\tau_{\text {nr }}$ decreased considerably (by $\sim 50 \%$ from the initial value of $\tau_{\mathrm{nr} 1} \approx 140 \mathrm{ps}$ at the same conditions). As seen in figure 2 , the decrease in $\tau_{\mathrm{nr} 1}$ (green triangles) and $\eta$ (black circles) proceeds with increasing $N$ at a similar rate. This is an indication that the photomodification enhances the nonradiative recombination, which in turn causes the decrease of the diffraction efficiency $\eta$.

Only a small decrease by $\sim 10 \%$ from its initial value $(270 \mathrm{ps})$ has been observed after photomodification in the slow grating decay time $\tau_{\text {eff2 }}$. This component is caused by localized carriers, so their ability to reach the nonradiative recombination centers formed by photomodification is limited. In addition, we conclude that photomodification has no strong influence on the localization conditions, since the photomodification does not change the PL band shape [6] or the temperature dependence of the PL peak position, as it was observed from PL measurements. Therefore, $\tau_{\mathrm{eff} 2}$ is weakly affected by the photomodification.

To estimate the effect of photomodification on the luminescence efficiency in AlGaN alloys, we measured the PL spectra and calculated the internal quantum efficiency (IQE) defined as IQE = $B N_{P L}^{2} / N_{P L} / \tau_{n r 1}+B N_{P L}^{2}$ using $\tau_{\text {nr1 }}$ and $B$ obtained from the LITG measurements, as described above.

The $\mathrm{Al}_{0.71} \mathrm{Ga}_{0.29} \mathrm{~N}$ epilayer for the PL characterization was photomodified with 1600 laser pulses of 4 ns duration at $N \approx 2 \times 10^{20} \mathrm{~cm}^{-3}$, matching the conditions of the modifying carrier density in LITG 
measurements. The PL intensity in $\mathrm{Al}_{0.71} \mathrm{Ga}_{0.29} \mathrm{~N}$ epilayer decreased by $\sim 40 \%$, approximately as much as $\tau_{\text {nrl }}$. This is an indication that PL intensity degraded due to the increase of the nonradiative recombination rate. A possible contribution from the light extraction change was estimated by comparing the decrease in the PL intensity and IQE. Within the experimental error, the drop for PL intensity and IQE was the same. Thus, the photomodification did not affect the light extraction.

The fact that the photomodification by short (25 ps) and long ( $4 \mathrm{~ns})$ pulses induces the same amount of changes leads to following implications about the duration of photomodification-induced processes. During the long pulse of $4 \mathrm{~ns}$ in the PL measurements, the carrier density exceeded the photomodification threshold for much longer time than during the short pulses in LITG. If the modification impact was proportional to the product of carrier density and duration, it would be much larger for long pulses (given the same modifying $N$ ); as a result, fewer long pulses would be needed to induce the same changes if compared to short ones. Since this assumption contradicts the experimental findings, it has to be expected that (i) the photomodification-induced processes are longer than picosecond and nanosecond pulses, and (ii) the photomodification impact is determined by the carrier density at the beginning of these processes. These assumptions imply that when started the photomodification-induced processes are not influenced by additional carriers injected into the area, until the next pulse arrives. Expected duration of these processes should be longer than $4 \mathrm{~ns}$ and shorter than the span between the subsequent laser pulses (100 ms). Such a long process can be related to atom rearrangements in the lattice, which is expected to be rather slow.

The measurements demonstrated that the changes in PL and $\eta$ due to the photomodification varied in the layers with different $\mathrm{Al}$ content. Figure 3 shows the dependence of the normalized diffraction efficiency $\eta$ on the photomodification duration (controlled by the number of laser pulses). The photomodification was performed at the same carrier density $N=2 \times 10^{20} \mathrm{~cm}^{-3}$ for all the samples and durations, while all $\eta$ values were measured at $N_{\text {ref }}=3 \times 10^{19} \mathrm{~cm}^{-3}$. The dependences depicted in figure 3 were sensitive to the Al content rather than to the potential profile characterized by the dispersions of the potential fluctuations $\sigma$. In particular, the diffraction efficiency in high-Al-content $\mathrm{Al}_{0.71} \mathrm{Ga}_{0.29} \mathrm{~N}$ decreases considerably faster than that in $\mathrm{Al}_{0.48} \mathrm{Ga}_{0.52} \mathrm{~N}$, while the values of $\sigma$ (estimated from the temperature dependences of the PL band peak position) were very close: $43 \mathrm{meV}$ and $45 \mathrm{meV}$. This conclusion is reasonable in view of the assumption that the initial fast decay of LITG is caused by the free carrier recombination, which is not affected by the localization. The observed anticorrelation between the excess energy and photomodification strength excluded the direct heating of the crystal lattice due to the excess energy of the photoexcited electron-hole pairs as a possible mechanism of the photomodification. For example, the excitation of $5.8 \mathrm{eV}$ used in our experiments corresponds to the excess energy of $0.7 \mathrm{eV}$ in $\mathrm{Al}_{0.71} \mathrm{Ga}_{0.29} \mathrm{~N}$ and $2.1 \mathrm{eV}$ in $\mathrm{Al}_{0.16} \mathrm{Ga}_{0.84} \mathrm{~N}$; however, a decrease of the diffraction efficiency by up to $\sim 60 \%$ was observed in the first sample, while no changes were detected in the second sample. The photomodification did not significantly depend on the lifetime of the free carriers. As seen in figure 3, the photomodification of the samples with similar 
Al contents of $48 \%$ and $46 \%$ but different nonradiative lifetimes $\left(\tau_{48 \%} / \tau_{46 \%} \approx 1.6\right)$ proceeded with increasing photomodification duration at the same rate (green triangles and red circles in figure 3). Since the lifetime is mainly determined by the density of the nonradiative recombination centers, this is an indication that the photomodification introduced additional nonradiative recombination centers rather than modified the centers already existing in the epilayer.

The most distinct feature of the photomodification is a strong dependence on the aluminum content. To show this dependence, we extracted the average slope of $\eta$ function by approximating it linearly on $\log$-log scale (the fits are not depicted in figure 3 to avoid overlapping). The inset in figure 3 shows this parameter as a function of the $\mathrm{Al}$ content. The trend is obvious, especially for the high aluminum content. Moreover, even the samples grown by using different techniques (MOCVD and MEMOCVD $^{\circledR}$ ) but containing a similar Al percentage (46\% and 48\%) exhibited identical photomodification behavior. Thus, the sensitivity of $\mathrm{AlGaN}$ to the photomodification mainly depends on the $\mathrm{Al}$ content.

The photomodification affected both the carrier diffusivity and lifetime. Figure 4 shows the diffusion coefficient $D$ (black squares) and carrier lifetime $\tau_{\text {effl }}$ (green circles) as the functions of the photomodification duration in the $\mathrm{Al}_{0.48} \mathrm{Ga}_{0.52} \mathrm{~N}$ epilayer. The epilayer was photomodified at the carrier density $N=2 \times 10^{20} \mathrm{~cm}^{-3}$, while the LITG measurements were performed at $N_{\text {ref }}=3 \times 10^{19} \mathrm{~cm}^{-3}$. Initially, the diffusion coefficient increased and reached its peak value of $2.9 \mathrm{~cm}^{2} / \mathrm{s}$, which was 2.4 times higher than that in unmodified sample, after 400 laser pulses of the photomodification. Under further exposure, the diffusion coefficient gradually declined. The lifetime, on the other hand, decreased monotonously from $110 \mathrm{ps}$ to $80 \mathrm{ps}$ during the entire duration of modification.

\section{Discussion}

At this stage of the research only a tentative physical explanation for the non-monotonous changes in diffusivity can be presented. The initial increase of $D$ can be associated with the re-organization of crystalline structure of the layer due to $\mathrm{Al}$ atoms migration.[11-13] This can lead to the enhancement of carrier mobility, at least along some directions in the crystal. The competing process decreasing the mobility and lifetime can be ascribed to the photogeneration of defects that act as carrier scattering and recombination centers. These defects might be similar to those introduced by thermal annealing [14] or ageing, $[15,16]$ especially by accelerated ageing of the AlGaN LEDs accomplished at high carrier densities $\left(\sim 10^{19} \mathrm{~cm}^{-3}\right)$, in contrast to low-carrier-density natural LED ageing at $\sim 10^{18} \mathrm{~cm}^{-3}$. Nitrogen vacancies $\mathrm{V}_{\mathrm{N}},[11,14,17-19]$ sites of out-diffused A1,[11-13] and oxides of Al or Ga [11,2023] claimed to be important for the defect formation during ageing or annealing of AlGaN alloys might also be responsible for the photomodification-induced formation of the nonradiative recombination centers.

The photomodification enhancement in $\mathrm{AlGaN}$ with increasing $\mathrm{Al}$ content can hardly be interpreted by larger energy delivered by the nonradiative recombination of a nonequilibrium electron-hole pair 
to the recombination enhanced defect reaction. Even at small Al contents, this energy (approximately equal to the band gap of $\mathrm{AlGaN}$ ) considerably exceeds the upper limit of all known activation energies $(\sim 2.5 \mathrm{eV})$ of the various defect reactions in III-V semiconductors. [4,24-28] Thus, the enhancement is directly related with the aluminum content. The observed correlation between the $\mathrm{Al}$ content and the photomodification efficiency can be explained either by the enhancement of the nonradiative recombination or by the introduction of the defects containing aluminum. Since the energy position of $\mathrm{V}_{\mathrm{N}}$ level in $\mathrm{AlGaN}$ shifts down into the band gap as the $\mathrm{Al}$ mole fraction increases the probability of nonradiative recombination via this defect grows as well.[11,17] Consequently, the enhancement of the nonradiative recombination by the light-induced formation of the nitrogen vacancies is stronger in $\mathrm{AlGaN}$ with higher $\mathrm{Al}$ content. On the other hand, a high propensity of $\mathrm{Al}$ towards oxidation $[11,24,29]$ might result in the Al oxide formation causing nonradiative recombination centers, and a high Al content is favorable for this process. The oxidation occurs with or without surface passivation,[20] with or without encapsulation,[27] and even in the $\mathrm{N}_{2}$ atmosphere,[22] presumably because of the inevitable contamination by trace amounts of oxygen during the growth of the alloy, or by transfer into the bulk of the crystal along dislocations.[21,27] The origin and mechanisms of the nonradiative recombination center formation created by photomodification need further investigation. The study of the photomodification is of special interest in view of the development of AlGaN-based UV LDs and high-power FETs, since high injected carrier densities are typical for these devices.

\section{Conclusions}

The photomodification of the AlGaN epilayers with $\mathrm{Al}$ content ranging from 16 to $71 \%$ was observed under the laser illumination generating carriers at the densities exceeding a certain threshold. During this modification, the high-density photoexcited carriers activated the additional nonradiative recombination centers, which permanently reduced the free carrier nonradiative lifetime and IQE. The deterioration of these parameters was most pronounced in the Al-rich AlGaN layers due to deeper energy levels or higher densities of the additionally generated nonradiative recombination centers. A short laser photomodification also permanently enhanced the diffusivity. An increase in the diffusion coefficient of up to 2.4 times has been achieved in $\mathrm{Al}_{0.48} \mathrm{Ga}_{0.52} \mathrm{~N}$ epilayer at a small $(13 \%)$ simultaneous decrease in the carrier lifetime. This effect is highly promising as a potential technique for improving the performance of high frequency electronic and optoelectronic devices (HEMTs, photodetectors, etc.).

\section{Acknowledgments}

The work at VU was funded by the European Social Fund under the Global Grant measure project VP1-3.1-ŠMM-07-K-02-014. The work at RPI was supported by the US Army Cooperative Research Agreement (Project Manager Dr. Meredith Reed). 


\section{References}

1 W.C. Burdett, O. Lopatiuk, A. Osinsky, S.J. Pearton, L. Chernyak, Superlattices Microstruct. 34 (2003) 55-62.

2 L. Chernyak, A. Osinsky, V. Fuflyigin, E.F. Schubert, Appl. Phys. Lett. 77 (2000) 875-7.

3 W. Burdett, A. Osinsky, V. Kotlyarov, P. Chow, A. Dabiran, L. Chernyak, Solid-State Electron. 47 (2003) 931-5.

4 D.V. Lang, Ann. Rev. Mater. Sci. 12 (1982) 377-98.

5 L.C. Kimerling, Solid-State Electron. 21 (1978) 1391-401.

6 T. Saxena, G. Tamulaitis, M. Shatalov, J. Yang, R. Gaska, M.S. Shur, J. Appl. Phys. 114 (2013) 203103.

7 H.J. Eichler, P. Gunter, D.W. Pohl, Laser-Induced Dynamic Grattings, Springer-Verlag, New York, 1986.

8 A. Miller, Transient grating studies of carrier diffusion and mobility in semiconductors, in: E. Garmire, A. Kost (Eds.), Nonlinear Optics in Semiconductors II, Semiconductors and Semimetals, vol. 59, Academic, New York, 1998, pp. 287-92.

9 P.G. Eliseev, P. Perlin, J. Lee, M. Osinski, Appl. Phys. Lett. 71 (1997) 569-71.

10 J.F. Muth, J.D. Brown, M.A.L. Johnson, Y. Zhonghai, R.M. Kolbas, J.W. Cook Jr., J.F. Schetzina, in: C. Kuo et al. (Eds.), Proc. MRS, vol. 537, Cambridge University Press, 1998, p. G5.2.

11 X.A. Cao, A.A. Syed, H. Piao, J. Appl. Phys. 105 (2009) 063707.

12 S. Sawyer, S.L. Rumyantsev, M.S. Shur, Solid-State Electron. 52 (2008) 968-72.

13 A. Pinos, S. Marcinkevičius, J. Yang, Y. Bilenko, M. Shatalov, R. Gaska, M.S. Shur, Appl. Phys. Lett. 95 (2009) 181914.

14 T. Hashizume, H. Hasegawa, Appl. Surf. Sci. 234 (2004) 387-94.

15 C. Hodges, N. Killat, S.W. Kaun, M.H. Wong, F. Gao, T. Palacios, U.K. Mishra, J.S. Speck, D. Wolverson, M. Kuball, Appl. Phys. Lett. 100 (2012) 112106.

16 M. Tapajna, R.J.T. Simms, Y. Pei, U.K. Mishra, M. Kuball, IEEE Electron Device Letters 31 (2010) 662-4.

17 A.A. Syed, X.A. Cao, J. Phys. D: Appl. Phys. 42 (2009) 195105.

18 A. Pinos, S. Marcinkevičius, M.S. Shur, J. Appl. Phys. 109 (2011) 103108.

19 A. Pinos, S. Marcinkevičius, J. Yang, R. Gaska, M. Shatalov, M.S. Shur, J. Appl. Phys. 108 (2010) 093113.

20 F. Gao, B. Lu, L. Li, S. Kaun, J.S. Speck, C.V. Thompson, T. Palacios, Appl. Phys. Lett. 99 (2011) 223506.

21 M. Tapajna, S.W. Kaun, M.H. Wong, F. Gao, T. Palacios, U.K. Mishra, J.S. Speck, M. Kuball, Appl. Phys. Lett. 99 (2011) 223501. 
22 M. Higashiwaki, S. Chowdhury, B.L. Swenson, U.K. Mishra, Appl. Phys. Lett. 97 (2010) 222104.

23 X. Qin, H. Dong, J. Kim, R.M. Wallace, Appl. Phys. Lett. 105 (2014) 141604.

24 J. Jimenez, C. R. Phys. 4 (2003) 663-73.

25 K. Maeda, M. Sato, A. Kubo, S. Takeuchi, J. Appl. Phys. 54 (1983) 161-8.

26 Y.L. Khait, J. Salzman, R. Beserman, Appl. Phys. Lett. 53 (1998) 2135-7.

27 F.A. Houle, D.L. Neiman, W.C. Tang, H.J. Rosen, J. Appl. Phys. 72 (1992) 3884-96.

28 L. Sugiura, J. Appl. Phys. 81 (1997) 1633-8.

29 S.A. Nikishin, B.A. Borisov, A. Chandolu, V.V. Kuryatkov, H. Temkin, M. Holtz, E.N. Mokhov, Y. Makarov, H. Helava, Appl. Phys. Lett. 85 (2004) 4355-7. 


\section{Figure captions}

Figure 1. Normalized LITG transients recorded at several diffraction grating spacings $\Lambda$ in $\mathrm{Al}_{0.48} \mathrm{Ga}_{0.52} \mathrm{~N}$ epilayer after photomodification by 400 laser pulses; the inset shows inverse initial decay time $1 / \tau_{\mathrm{G} 1}$ as a function of inverse grating period $1 / \Lambda^{2}$ for simultaneous determination of nonequilibrium carrier diffusion coefficient $D$ and lifetime $\tau_{\text {effl }}$.

Figure 2. Dependences of normalized diffraction efficiency $\eta$ and nonradiative lifetime $\tau_{\text {nr } 1}$ on the density $N$ of nonequilibrium carriers generated during photomodification of $\mathrm{Al}_{0.71} \mathrm{Ga}_{0.29} \mathrm{~N}$ epilayer with 1600 laser pulses.

Figure 3. Normalized diffraction efficiency $\eta$ as a function of photomodification duration (number of laser pulses) in $\mathrm{AlGaN}$ epilayers with different $\mathrm{Al}$ content; in the inset: slope of this function (in log$\log$ scale) as a function of $\mathrm{Al}$ content.

Figure 4. Diffusion coefficient (black squares) and effective carrier lifetime $\tau_{\text {effl }}$ (green circles) as a function of photomodification duration (number of laser pulses) in $\mathrm{Al}_{0.48} \mathrm{Ga}_{0.52} \mathrm{~N}$ epilayer. 